\title{
Starting the Stereotactic Radiosurgery Facility in Bangladesh: An Initial Experience
}

\author{
Narendra Kumar ${ }^{1}$ Murugan Appasamy ${ }^{1} \quad$ Sania Ahsan $^{2} \quad$ Sandip Kumar Das ${ }^{3}$ Taohida Yasmin ${ }^{1}$ \\ K.M. Masud Rana ${ }^{1} \quad$ R. Arun Kumar ${ }^{1} \quad$ Aliuzzaman Joardar ${ }^{4} \quad$ Zillur Rahman $^{4} \quad$ Amitabha Chanda $^{4}$ \\ Saiful Islam Chapal ${ }^{5}$
}

${ }^{1}$ Department of Radiation Oncology, Evercare Hospital Dhaka, Dhaka, Bangladesh

${ }^{2}$ Department of Radiodiagnosis, Evercare Hospital Dhaka, Dhaka, Bangladesh

${ }^{3}$ Department of Neurology, Evercare Hospital Dhaka, Dhaka, Bangladesh

${ }^{4}$ Department of Neurosurgery, Evercare Hospital Dhaka, Dhaka, Bangladesh

${ }^{5}$ Department of Neurosurgery, National Institute of Neurosciences \& Hospital, Dhaka, Bangladesh

\author{
Address for correspondence Narendra Kumar, MD, MAMS, \\ Department of Radiation Oncology, Evercare Hospital Dhaka, \\ Plot 81 Block E, Dhaka 1229, Bangladesh \\ (e-mail: drnarendra74@gmail.com).
}

\begin{abstract}
Keywords

- agility

- intracranial

- linear accelerator

- stereotactic localizer box

- stereotactic radiosurgery

Radiotherapy is an integral part of a well-defined cancer management program. Bangladesh is a fast-growing East Asian country with 161 million population, and approximately 2 lakh cancer patients are diagnosed newly ever year. Though there are several modern treatment facilities to deliver radiotherapy, only limited facilities were available to deliver stereotactic radiotherapy (SRS) programs for needy patients. Evercare Hospitals Dhaka has recently started SRS and stereotactic therapy (SRT) with a dedicated team of trained people. The hospital is equipped with modern linear accelerator capable of delivering high dose rate mode and micro multileaf collimators with $2.45-\mathrm{mm}$ thickness at isocenter to deliver larger doses of radiation precisely. This paper summarizes patient selection criteria and workflow for the SRS/SRT treatment established, including patient preparation, image data acquisition, target and organ at risk delineation on CT (computed tomography) and MRI (magnetic resonance imaging) images, treatment planning process, and quality assurance. Each stage of the process is explained in detail, with specific emphasis on certain areas to achieve a higher degree of accuracy. This article also highlights the need for dedicated timeout procedures to be followed to avoid drastic errors in treatment delivery. We also summarized demographics data of patients treated in the first 6 months, including diagnosis, sizes of lesions, and dose fraction. The dosimetric and setup uncertainties encountered during SRS/SRT treatment delivery are also described. Though, initially, we faced challenges, SRS/SRT treatment was successfully implemented and more than 35 patients were treated, with appreciable clinical outcomes.
\end{abstract}

published online Jun 13, 2020
DOI https://doi.org/

$10.1055 / \mathrm{s}-0040-1716814$ ISSN 2454-6798. (c) 2020. Spring Hope Cancer Foundation \& Young Oncologist Group of Asia.

This is an open access article published by Thieme under the terms of the Creative Commons Attribution-NonDerivative-NonCommercial-License, permitting copying and reproduction so long as the original work is given appropriate credit. Contents may not be used for commercial purposes, or adapted, remixed, transformed or built upon. (https://creativecommons.org/licenses/by-nc-nd/4.0/) Thieme Medical and Scientific Publishers Pvt. Ltd., A-12, 2nd Floor, Sector 2, Noida-201301 UP, India 


\section{Introduction}

Radiotherapy plays an essential role in cancer management, and more than $60 \%$ of cancer patients require radiation as part of their cancer management program. As far as Bangladesh is concerned, there are 13 to 15 lakh cancer patients in Bangladesh, with approximately 2 lakh patients newly diagnosed with cancer each year. ${ }^{1}$ Increasing population and longevity are leading to an increasing number of cancer patients in Bangladesh. When combined with population aging, the increase in cancer prevalence is inevitable. ${ }^{2}$ The availability of a radiation facility is still suboptimal. There are 22 linear accelerators, 12 telecobalt units, and 10 high dose rate (HDR) brachytherapy units in entire Bangladesh. Out of all available accelerators, till the date, only our center is equipped enough to deliver stereotactic radiosurgery (SRS). Hence, to avail SRS treatment, patients have to go out of the country. We have state-of-the-art Elekta Versa HD linear accelerators with Apex Dynamic mMLC, which makes it competent to deliver SRS for all possible indications (malignant, nonmalignant, and functional). SRS technique is a procedure that delivers high doses of radiation precisely to the targeted lesion in only a single or few sessions when compared with conventional radiation therapy. In the case of SRS, higher doses are given in multiple fractions. The goal of SRS/stereotactic therapy (SRT) is to deliver very high radiation doses that will destroy the lesion/tumor and achieve permanent local control. SRS is an equally effective alternative to invasive surgery, especially for patients who are unable to undergo surgery and for tumors and abnormalities that are hard to reach by open surgery, those located close to vital organs/anatomical regions, or residual tumors/lesions after surgery. ${ }^{3}$

Evercare Hospitals Dhaka (formerly Apollo Hospitals Dhaka) foresees to establish a state-of-the-art radiotherapy facility at par with world standard in Dhaka and started a comprehensive cancer care facility combined with PET-CT (positron emission tomography-computed tomography) imaging since September 2017. We began with radiation techniques such as intensity-modulated radiotherapy and volumetric-modulated arc therapy (VMAT) in combination with HDR brachytherapy. In early 2019, we decided to start SRS for intracranial lesions in Bangladesh. The first case of SRS at Evercare Hospital was arteriovenous malformation (AVM), which was treated in April 2019.

This article aims to share our experience of setting up of an SRS facility in this country, which includes joining hands with different disciples, educating them about their needs, explaining their role, acquiring required accessories, educating the radiotherapy team about the importance of accuracy of the procedure, patient selection criteria, patient assessment, patient preparation, simulation, and planning, quality checks, and treatment delivery.

\section{Patient Selection}

Following are the common indications for SRS:

1. Brain tumors including benign and malignant tumors (e.g., pituitary adenoma, meningiomas, trigeminal schwannomas, vestibular schwannomas, chordomas, craniopharyngioma, gliomas), brain metastasis, single or multiple, residual tumor following surgery;

2. AVMs, which are tangles of expanded blood vessels that disrupt normal blood flow in the brain and sometimes bleeds, cavernoma;

3. Other neurological indications, e.g., trigeminal neuralgia (a nerve disorder in the face), hypophysectomy, thalamotomy, tremor, etc.

\section{Radiobiology of SRS}

The highly ablative potential of SRS is because of its radiobiological effectiveness. A single dose of 12 to $25 \mathrm{~Gy}$ of radiation leads to direct double-strand DNA break overcoming hypoxia. Second, it indirectly kills the resistant perivasculature tumor stem cell niche through vascular endothelial disruption and immune response, which act through cytotoxic T cells. As a result, these cells lose their ability to reproduce. After SRS, benign tumors usually shrink over 18 months to 2 years. Malignant and metastatic tumors may shrink more rapidly, within a couple of months. AVMs may begin to thicken and close off slowly over several years following treatment. Many tumors will remain stable and inactive without any change. Since the aim is to prevent tumor growth, this is considered a success.

\section{Initial Assessment and Consultation}

Patients referred to us for SRS, first complete the registration process with demographic information. Nursing assessment is done by recording patient's vitals, fall risk scoring by modified Morse scale, and pain assessment using Wong Baker Scale/FLACCS scale by nursing staff. A comprehensive evaluation of patients was done by reviewing previous treatment records, available imaging studies, general physical examination, and detailed neurological assessment. Complete investigations including imaging are performed as required to assess patients' eligibility to undergo SRS. Patients and family members are educated in detail about the role of SRS and possible outcomes in their case and about steps of the SRS with a brochure. We also explain possible alternate options, if any. Furthermore, based on lesion size/type/location/stage, patients' performance status, and compliance, we decide the appropriate SRS dose and fractionation, and, if needed, a multidisciplinary tumor board is constituted beforehand. Once decided for SRS, informed written consent is obtained from the patient and family members, explaining the indication of treatment, outcomes, alternate treatment options, possible risks, complications, side effects, and long-term consequences of the procedure.

\section{Patient Preparation}

After explaining the patient about the entire process and importance of his/her co-operation throughout the procedure, we do the patient preparation on the actual linear accelerator couch to achieve a higher degree of accuracy in 
the preparation of the SRS/SRT patient mask. First, the SRS/ SRT head frame holder is attached to the linear accelerator with the headrest holder to hold the patient head during treatment rigidly. This unique farm acts as a base for attaching the special thermoplastic mask. It has provisions to attach the stereotactic localization box and to attach the mouth bit for patients if required. It has special adjustment screws on its bottom to adjust the sag of the headrest holder, depending on the patient's head weight.

After that, the patient is asked to lie down on the couch comfortably, and the patient's head is adjusted to be in line with the sagittal laser of the linear accelerator. A customizable small vacuum cushion is kept as a headrest to position the patient on the frame. If needed, the frame level is adjusted with the help of reference lines in the localization box using screws, as mentioned previously. Once the position is finalized, the thermoplastic mask is prepared with the same position. If needed, laser reference marks are drawn on the thermoplastic mask to reproduce and cross-check the positioning. As part of our radiotherapy process, the accessories used for the patient are recorded in detail on a setup sheet, and, if needed, photos are taken to reproduce the position (-Fig. 1A, B).

\section{Image Acquisition and Target Delineation}

To acquire the computed tomographic (CT) images of the patient, the patient is positioned on a CT scanner connected to a treatment planning system (TPS). The patient's position is reproduced as the patient was placed during preparation, and a stereotactic localizer box is also inserted around the patient's head. CT images are acquired with thin slice thickness, which is $1.25 \mathrm{~mm}$ in our case. The field of view (FOV) of scout scan is kept sufficiently large to cover the localizer box because this box has markers on two lateral and anterior sides and can be seen in the axial slices of the CT image. It helps to locate the stereotactic coordinated of the target. The CT images were acquired with contrast using two series, an immediate and a delayed (4-6 minutes), as per the diagnosis to enhance the tumor's visibility (-Fig. 2).

A contrast magnetic resonance imaging (MRI) of the patient is also acquired with a thin slice thickness (preferably $1 \mathrm{~mm}$ ) for the entire brain region, starting from the vertex to the C2 level. During the MRI scan, all the accessories used for positioning patients in CT cannot be used due to its compatibility, except the vacuum cushion headrest. Still, it is not a problem, and the patient position can be adjusted during the image fusion process (-Fig. 3 ).

All the acquired images are transferred to the Monaco TPS (Elekta), and the CT and MRI images are fused using the TPS. The accuracy of the fusion was checked by comparing the anatomical landmarks in the brain, such as the skull, eyeballs,

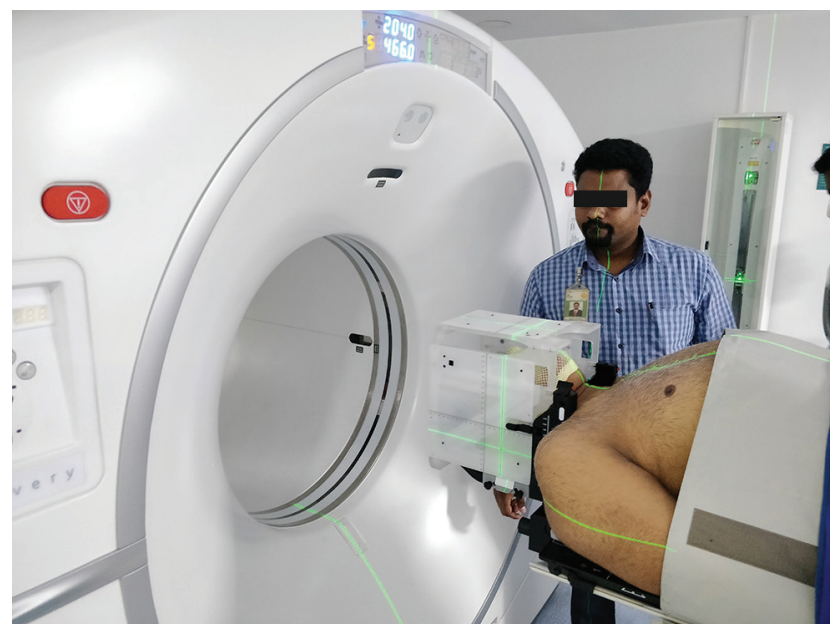

Fig. 2 Acquiring CT (computed tomography) simulation image with the stereotactic localizer box.

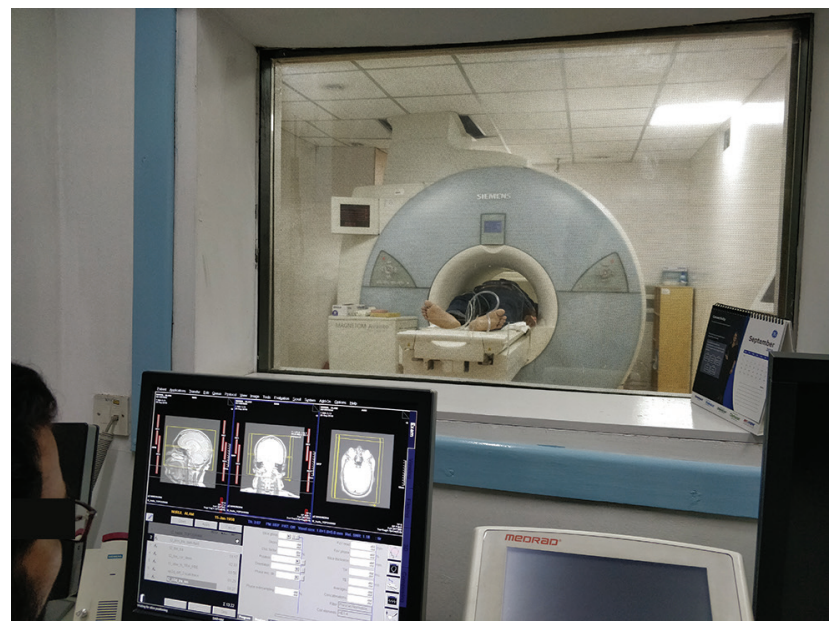

Fig. 3 View from the MRI (magnetic resonance imaging) control console during imaging.
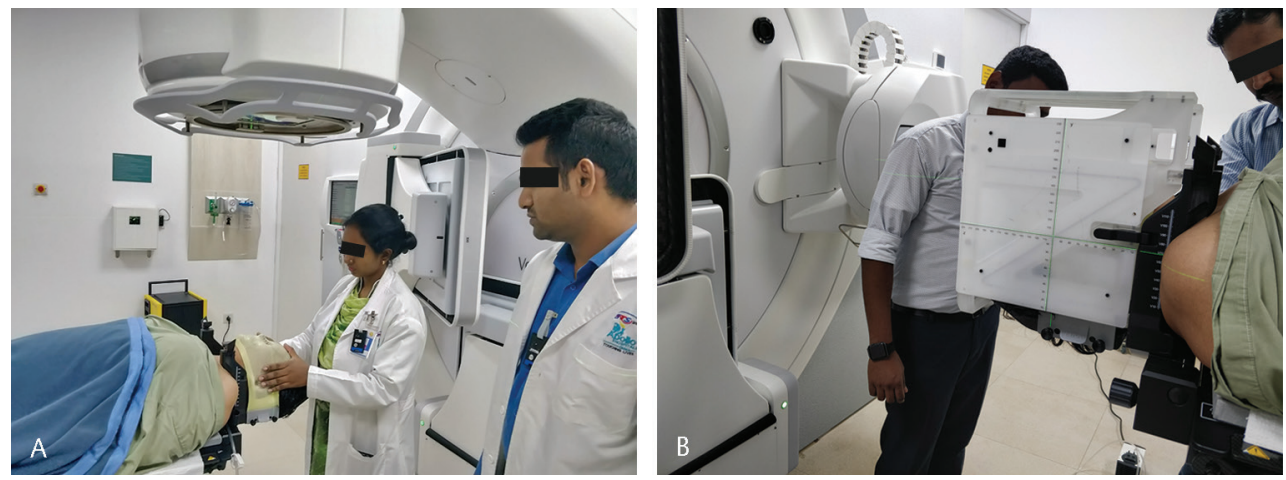

Fig. 1 (A) Preparation of the stereotactic radiotherapy (SRS) mask on the headrest attached to the treatment couch. (B) SRS mask with the stereotactic localizer box. 
and pituitary cavity,. by an experienced medical physicist and radiation oncologist before starting the contouring process. ${ }^{4}$ The contouring is comprehensively performed by following the standard guidelines for all organs at risk (OARs) and target volumes, including gross tumor volume (GTV) and planning target volume (PTV), by an experienced radiation oncologist. The GTV delineation is finalized after a consensus among radiation oncologists, neurosurgeons, and neuroradiologist. ${ }^{5}$ Appropriate target dose and dose constraints are prescribed and then handed over to a medical physicist for generating a plan ( - Fig. 4A, B).

\section{Treatment Planning and Evaluation}

A medical physicist generates a radiation treatment plan. The treatment plan aims to deliver the maximum dose to the target and keep the dose to surrounding critical structure within an acceptable limit. Depending on the size of the target and location, the delivery method is selected, either a dynamic conformal arc by a micro multileaf collimator (micro-MLC) or volumetric-modulated arc therapy (VMAT) with an Agility collimator (Elekta). In the case of micro-MLC, we use the 2.46-mm-thick APEX collimator for delivering radiation, whereas, in the case of VMAT, the regular Agility collimator with a 5-mm-thick MLC is used. The dynamic arc delivery is the method of treatment, in which the micro-MLC takes the shape of the target continuously while the gantry moves around the patient, the micro-MLC will move dynamically depending on the nearby critical structures. Still, its ability to modulate the intensity of the radiation beam is limited; hence, it is not a suitable method for tumors located near a critical structure or overlapping with the target. And it is ideal for cases with isolated targets and those situated in the peripheral areas. The VMAT technique is preferable in targets located near a critical structure. It gives us the freedom to escalate the dose and the ability to modulate the radiation dose according to the clinical needs.

Mostly, noncoplanner partial arcs are used as per the standard guideline for the treatment planning purpose. These partial arcs help contain the low-dose spread as well as increase the coverage of the target. The Monte Carlo based algorithm is used for the dose calculation to achieve higher dose calculation accuracy, and the 6-MV flattening filter-free (FFF) mode is the preferred choice to achieve minimal treatment time as it has the ability to deliver $1,800 \mathrm{MU} /$ minute $(1,800 \mathrm{cGy} /$ minute). ${ }^{6,7}$ Inverse treatment planning optimization method will be adopted to achieve the required coverage to the target and at the same time importance is given to the OARs, and the Radiation Therapy Oncology Group (RTOG) SRS/SRT dose constraints are used to restrict dose to OARs during optimization. All possible efforts are made to reduce the integral dose and to increase the dose escalation to achieve the adequate coverage to target volume as per the prescription and to have control over the hot areas inside the target volume. A deliverable plan is chosen for evaluation with radiation oncologists only if the coverage is adequate, that is, 85 to $95 \%$ of the target covers 95 to $100 \%$ of prescription, and all OARs doses were well within the RTOG criteria. The optimization and planning process are repeated until we achieve the preceding goal.

The chosen plan is jointly evaluated by a physicist and a radiation oncologist following standard guidelines for its suitability to deliver to the patient. The target coverage and hot spots are evaluated in dose-volume histogram (DVH) as well as in cross-sectional views (axial, sagittal, and coronal), preferably with a color wash. In the case of SRS/SRT, up to 120 to $130 \%$ of the hot spots are also acceptable in some instances, depending on clinical requirements. No compromises were made if there was any spill of dose outside the target volume and discrepancies inadequate dose coverage to target. The OAR dose is evaluated against the RTOG dose constrains both in DVH and in isodose distribution views. Once the plan is accepted, the plan is sent to the MOSAQ record and verification system to prepare the plan for delivery. Beforehand, all the treatment planning parameters specific to the patient, including prescription dose, fraction size if any, number of beam or arc, beam or arc angle, collimator angle if any, couch rotation angle, beam or arc direction and sequence in accordance with couch rotation to avoid the collision, number of segments, arc direction, and the total number of monitor units for each beam, are recorded as per the department protocol. All the documents are signed after being rigorously checked by both a medical physicist and a radiation oncologist, and a second check by another medical physicist is mandatory as per our department FMEA (Failure Mode and Effect Analysis) protocol to avoid any potential error. The treatment plan is also checked by a radiotherapy technologist for its deliverability ( - Fig. 5).
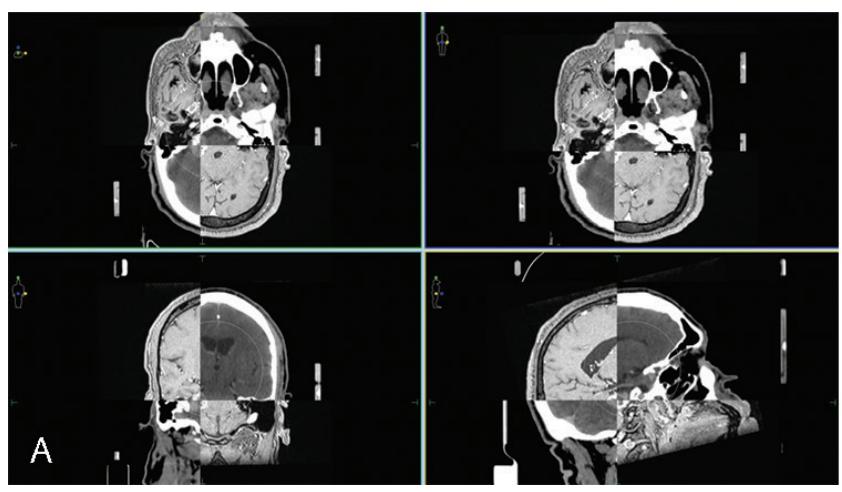

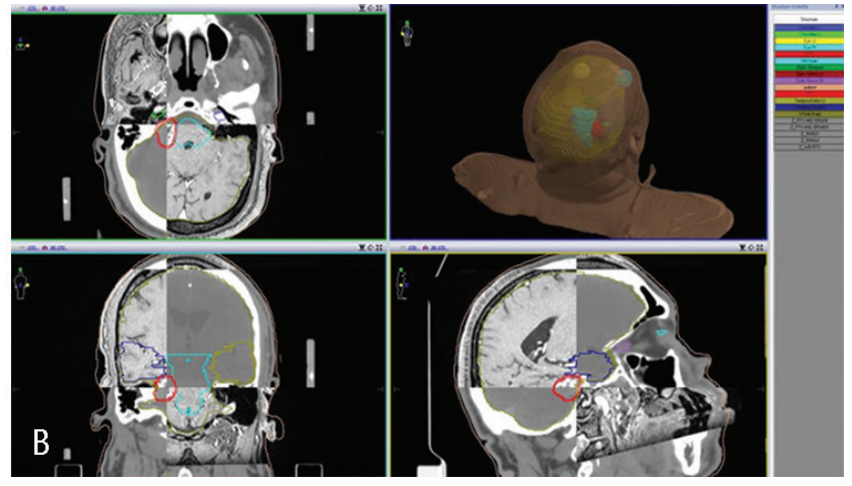

Fig. 4 (A) CT-MRI fusion. (B) Target volume and organ-at-risk delineation. CT-MRI, computed tomography-magnetic resonance imaging. 


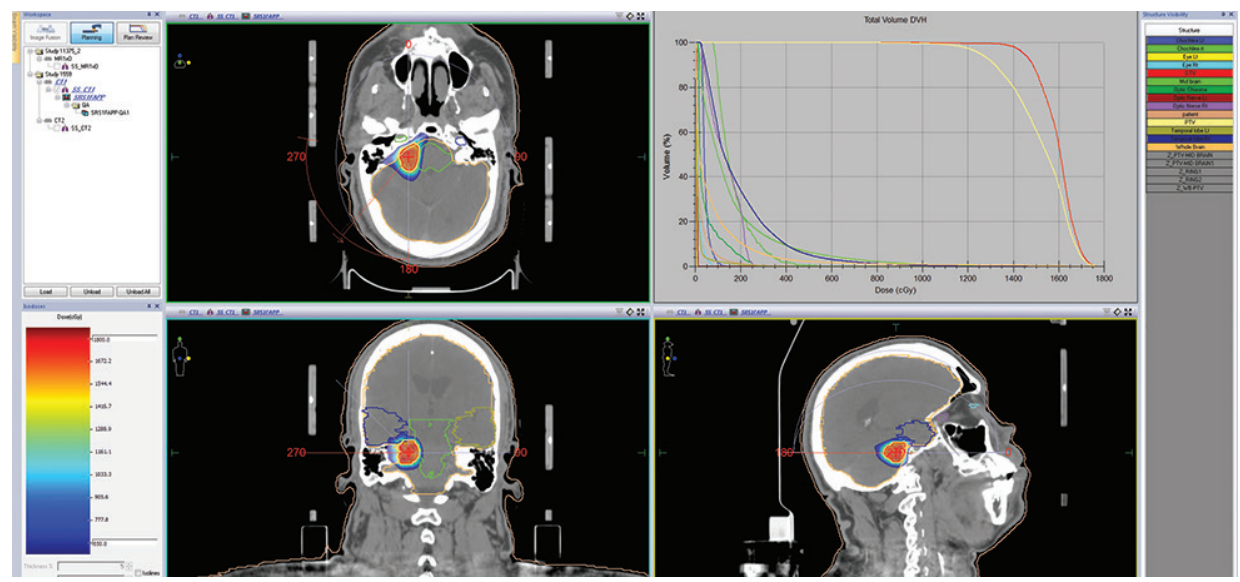

Fig. 5 The treatment plan showing radiation dose distribution in colorwash and dose-volume histogram.

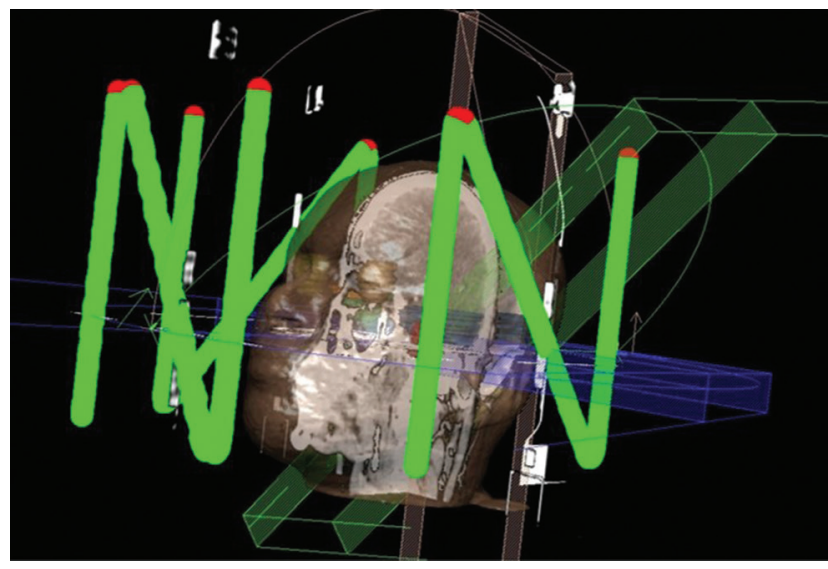

Fig. 6 Digitized "Z" shaped marker on the localizer box.

\section{Obtaining Stereotactic Shift using the Localizer}

The finalized plan is then transferred to a particular software called "Locate," in which the "Z" shaped frame in the localizer box, as mentioned earlier, will be digitized with the help of markers visible in each axial slice. The process is time-consuming and needs to be performed accurately, as if there is any error, then the error will be added to the overall uncertainty. After digitization, the stereotactic shift of the plan isocenter can be obtained using the software. The shift can be printed out in the form of "Target sheets," in which the isocenter coordinated will be marked. Those shifts will be in millimeters. The target sheet can also be inserted in the localizer box to ease the setup process (-Fig. 6).

\section{Patient-Specific Quality Assurance Check}

A patient-specific quality assurance (QA) test is mandatory. It needs to meet the pass criteria for all the high precision radiotherapy treatments as per the ICRU (International Commission on Radiation Units and Measurements) ${ }^{8}$ recommendation before it is delivered to the patient. The approved plan is transferred to a QA phantom specific for patient QA; we, in our center, utilize the IBA Imattrix FFF phantom for this purpose. This Imattrix FFF array is designed specially to yield accurate results even at very high dose rates in the FFF mode. A sample of the dose plan from the phantom is taken from a predetermined coronal plan at the isocenter position and is used as a calculated dose. The phantom is then irradiated with the approved treatment plan after setting up the Imattrix FFF in the QA setup on the linear accelerator. The delivered dose is recorded with the help of my QA Imattrix FFF software, and a sample of coronal dose plan is obtained in the same position and used as a measured dose. The calculated dose obtained from TPS and the measured dose obtained from QA phantom are compared using the my QA patient IBA software, and the gamma index values are calculated. It should pass a minimum of $90 \%$ for $3 \%$ and $2-\mathrm{mm}$ distance to an agreement value in order to accept the plan to be eligible to deliver to the patient. In our case, all our patients showed $98 \%$ pass results.

\section{Pretreatment Checks}

For all the high-precision radiotherapy patients, before the patient is scheduled for first-day treatment, all parameters related to patient identity, positioning devices, and a treatment plan, including availability of required accessories, beam or arc angle, number of beam or arc, collimator angle, couch angle, number of monitor units (MU), the sequence of beams or arcs, and its suitability for collision-free delivery and radiation dose prescription with authorized person's signatures, are checked by a radiotherapy technologist. Also, all related documents, including setup sheets, treatment planning sheets, completely filled radiotherapy treatment charts, daily patient assessment notes by nurses, and registers are checked before the patient is called for the treatment. As part of the Joint Commission International standard guideline, all our radiotherapy patients undergo a detailed nursing assessment before every radiotherapy treatment. A doctor also performs a posttreatment check before the patient is sent from the department. Similarly, all the dosimetric parameters are also checked by a second physicist as part of our standard protocol. A dummy run is performed by a senior technologist 
and medical physicist to enable interruption-free treatment delivery for the patient once everything is ready. The appropriate correction would be applied immediately if any errors/ collisions are found.

\section{Treatment Execution}

Before taking the patient on the treatment couch, vitals are measured by staff nurse and his/her identity is verified as per the International Patient Safety Guidelines, for example, photo ID card and universal patient identity number. After checking the availability of necessary equipment for the patient, he/she is taken to the treatment room accompanied

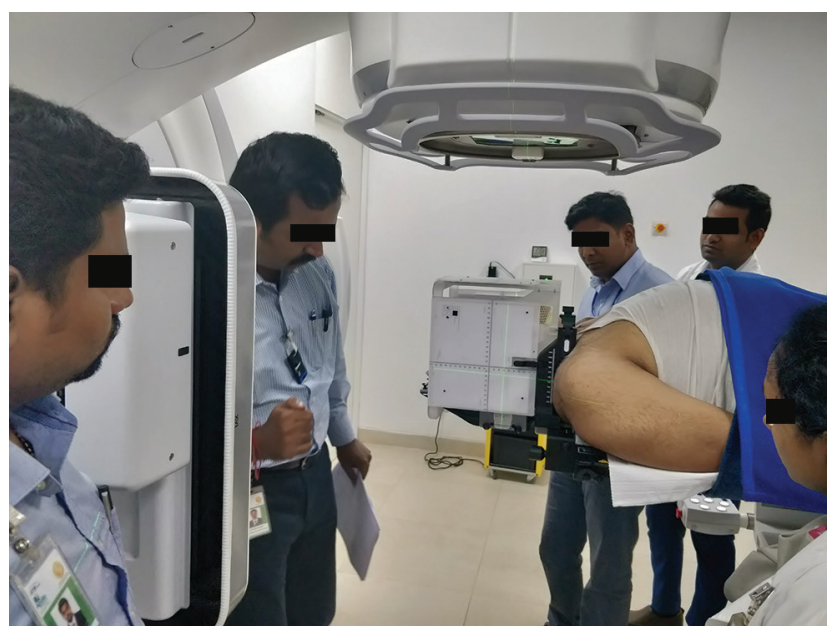

Fig. 7 Patient positioned on the treatment couch and applied stereotactic shift just before acquiring the $\mathrm{CBCT}$ image. $\mathrm{CBCT}$, cone beam computed tomography. by a patient care attendant. The patient is positioned on the treatment couch as per the setup sheet. Radiation oncologists and technologists verify the patient's position. With the help of a stereotactic localizer, the required stereotactic shift is applied as per the target sheet printout values ( - Fig. 7 ). The on-board kVCBCT (kilo-voltage cone beam CT) scan of the patient is acquired to verify the treatment setup using the $\mathrm{kVCT}$ attached to the linear accelerator. In this process, the patient CT scan is acquired just before the treatment coregistered with the originally obtained planning CT images, and the patient positioning shift is calculated by matching the gray values (similar to $\mathrm{HU}$ value of CT images) of each pixel of the both CT image series. The shift can be calculated to the millimeter level and can be corrected accordingly before the start of treatment. ${ }^{9}$ In the case of SRS/SRT treatment, the repeated CBCT images are acquired and correction is applied till we get almost zero shift in all directions, the rotational correction can also be addressed because of the six-dimensional robotic couch attached the linear accelerator ( - Fig. 8).

After verifying the patient's position, a dedicated time out will be performed just before switching ON the radiation beam to verify patient identity, diagnosis, treatment modality, energy used, daily dose, number of fractions, adoption of special instructions, and number of monitor units. The timeout is led by a senior radiotherapy technologist. The entire team participates in the procedure, including treating radiation oncologist, radiation oncology register, and medical physicist, and the treatment is delivered only upon agreement of all members. During the treatment execution, the patient is monitored with closed-circuit television (CCTV), and any two-way voice communication.

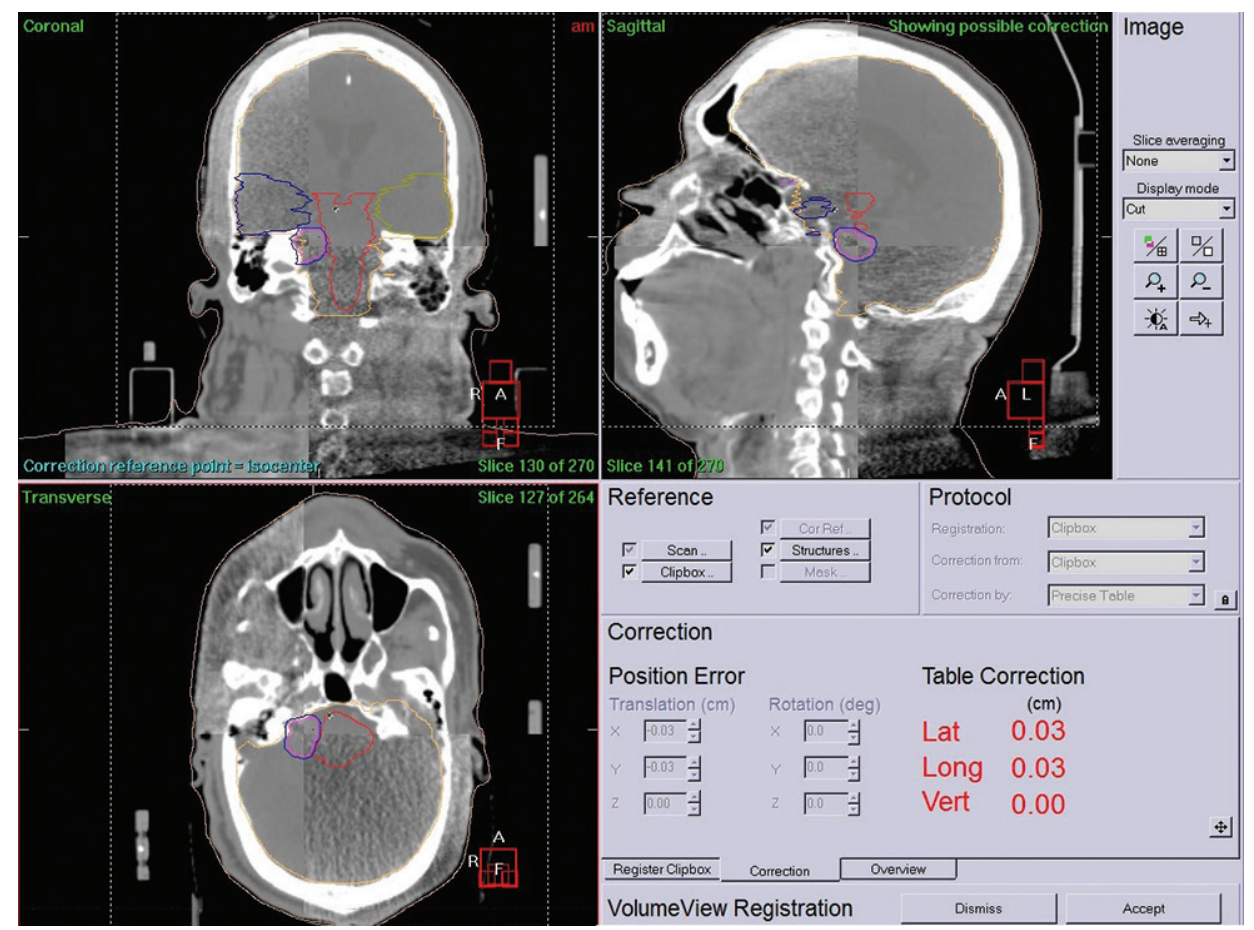

Fig. 8 CBCT fused with planning $C T$ to assure submillimeter accuracy just before treatment. CBCT, cone beam computed tomography. 


\section{Patient Statistics}

Table 1 summarizes the number of patients treated in the first 6 months since the introduction of this modern facility in our department, till now we have treated more than 35 patients. The patient's age ranged from 25 years up to 75 years. Most of the patients were treated with the 6-MV FFF mode using the VMAT delivery method. The radiation dose ranged from 13 to $25 \mathrm{~Gy}$ for benign lesions and may go up to as high as 90 to $120 \mathrm{~Gy}$ for functional diagnosis. Most of our patients were followed up for a period of 6 to 9 months. Clinical outcomes show a promising result, and most of our patients are able to maintain their routine life without any significant complications (-Table 1).

\section{Initial Challenges}

When we started discussing, initially, about the possibility of introducing the SRS/SRT treatment facility, the major concern was about getting the appropriate patients. Though the country has several eligible patients who require SRS or SRT, most of the patients do not have enough confidence to undergo this type of high precision treatment in the country. They believe that even if it is being done in this country, it will not be at par with the world's standard. Hence, the first challenge was to break the conventional thought of people and about their misconception and their underestimation about the hospitals in Bangladesh. To achieve that, we initially started participating in many scientific events, in our hospital and across Dhaka, and educated/convinced our medical community about the versatile technology of our SRS/SRT treatment facility provided by Elekta and efficiency/knowledge of our team. We also started meeting our neurology and radiology colleagues. We started explaining to them about the cutting-edge technology we got at Evercare Hospital for SRS/SRT treatment. We also arranged some departmental tours for some targeted senior people. With all the hard work after a few months, the first patient referred to us for SRS was an AVM, in April 2019.

After successfully treating many patients, we, with our team, spread the message across the country so that the needy patients can avail this start-of-the-art facility available in the country, and now people of this country can avoid traveling abroad for this high precision radiotherapy.

Table 1 Patients characteristics and dosimetric indices with positional accuracy during patient treatment

\begin{tabular}{|c|c|c|c|c|c|c|c|c|c|}
\hline \multirow[t]{3}{*}{ S. no. } & \multicolumn{4}{|c|}{ Patients characteristics } & \multicolumn{5}{|c|}{ Dosimetric parameter } \\
\hline & \multirow[t]{2}{*}{ Age/sex } & \multirow[t]{2}{*}{ Diagnosis } & \multirow[t]{2}{*}{ Volume } & \multirow[t]{2}{*}{ Dose } & \multirow[t]{2}{*}{$\begin{array}{l}\text { Heterogeneity } \\
\text { index }\end{array}$} & \multirow[t]{2}{*}{$\begin{array}{l}\text { Conformity } \\
\text { index }\end{array}$} & \multicolumn{3}{|c|}{$\begin{array}{l}\text { Accuracy of } \\
\text { execution }\end{array}$} \\
\hline & & & & & & & $\mathrm{X}$ & $\mathrm{Y}$ & Z \\
\hline 1 & $24 / \mathrm{M}$ & AVM & 39.7 & $23 / 2 \#$ & 1.41 & 0.83 & 0.2 & -0.1 & 0.0 \\
\hline 2 & $57 / \mathrm{M}$ & $\begin{array}{l}\text { Trigeminal } \\
\text { schwannoma }\end{array}$ & 13.8 & $14 / 2 \#$ & 1.38 & 0.81 & -0.2 & -0.1 & -0.2 \\
\hline 3 & $47 / F$ & $\begin{array}{l}\text { Trigeminal } \\
\text { schwannoma }\end{array}$ & 11.2 & $13 / 2 \#$ & 1.37 & 0.62 & 0.3 & 0.2 & 0.4 \\
\hline 4 & $61 / \mathrm{M}$ & $\begin{array}{l}\text { Trigeminal } \\
\text { neuralgia }\end{array}$ & 0.109 & $90 / 1$ & 1.13 & 0.53 & 0.9 & 0.4 & 0.1 \\
\hline 5 & $68 / \mathrm{M}$ & Brain metastasis & $\begin{array}{l}41 \\
\text { and } 11.3\end{array}$ & $18 / 1$ & $1.04,1.06$ & $0.58,0.57$ & 0.7 & 0.1 & 0.1 \\
\hline 6 & $74 / F$ & Brain metastasis & $\begin{array}{l}0.36, \\
0.6,1.7 \\
\text { and } 4.2\end{array}$ & $24 / 1$ & $\begin{array}{l}1.2,1.16,1.14 \text {, } \\
\text { and } 1.31\end{array}$ & NA & 0 & 1.1 & 0.2 \\
\hline 7 & $41 / F$ & CNS lymphoma & 8.5 & $16 / 1$ & 1.17 & 0.81 & -0.1 & 0.1 & -0.3 \\
\hline 8 & $60 / F$ & Cavernoma & 1.5 & $14 / 1$ & 1.24 & 0.74 & -0.4 & 0.7 & -1.4 \\
\hline 9 & $60 / \mathrm{F}$ & Brain metastasis & $\begin{array}{l}0.54,3.4, \\
\text { and } 17.1\end{array}$ & $22 / 1,24 / 1,15 / 1$ & $\begin{array}{l}1.13,1.13, \\
\text { and } 1.37\end{array}$ & $\begin{array}{l}0.60,0.24 \text {, and } \\
0.39\end{array}$ & 0.1 & 0.3 & -0.2 \\
\hline 10 & $46 / F$ & Brain metastasis & $\begin{array}{l}0.67,1.2, \\
\text { and } 14.3\end{array}$ & $14 / 1,18 / 1,14 / 1$ & $\begin{array}{l}1.33,1.21 \text {, } \\
\text { and } 1.15\end{array}$ & $\begin{array}{l}0.67,0.38 \text {, and } \\
0.02\end{array}$ & -0.1 & 0 & -0.1 \\
\hline 11 & $63 / M$ & Brain metastasis & $\begin{array}{l}0.4,0.54 \text {, } \\
\text { and } 9.5\end{array}$ & $24 / 1$ & $\begin{array}{l}\text { 1.09, 1.16, } \\
\text { and 1.15 }\end{array}$ & $\begin{array}{l}0.64,0.06 \text {, and } \\
0.03\end{array}$ & -0.2 & -0.4 & -0.2 \\
\hline 12 & $62 / \mathrm{M}$ & $\begin{array}{l}\text { Vestibular } \\
\text { neuroma }\end{array}$ & 6.6 & $13 / 1$ & 1.35 & 0.80 & -0.3 & -0.3 & 0 \\
\hline 13 & $40 / \mathrm{M}$ & Meningioma & 4.5 & $13 / 1$ & 1.25 & 0.65 & 0.3 & 0.1 & 0.2 \\
\hline 14 & $53 / \mathrm{M}$ & Brain metastasis & 19.16 & $24 / 1$ & 1.14 & 0.89 & 0 & -0.1 & 0.2 \\
\hline 15 & $55 / \mathrm{M}$ & Brain metastasis & 11.05 & $22 / 1$ & 1.21 & 0.89 & -0.6 & 0.0 & 0.3 \\
\hline 16 & $38 / \mathrm{M}$ & $\begin{array}{l}\text { Vestibular } \\
\text { schwannoma }\end{array}$ & 62.4 & $18 / 3$ & 1.07 & 0.93 & 0.6 & 0.1 & 1.1 \\
\hline
\end{tabular}

Abbreviations: AVM, arteriovenous malformation; CNS, central nervous system; F, female; M, male. 
We went across the country, including major cities, major medical colleges, scientific associations, national institutes, and different targeted groups of medical practitioners, to spread the news of the availability of world standard SRS/SRT facility at their doorstep.

\section{Summary}

SRS is a well-established modality to treat various malignant, nonmalignant, and functional intracranial lesions. ${ }^{8}$ The recent advancement in radiotherapy treatment delivery technology such as smaller MLCs and high dose rate modes (FFF mode) gives more freedom to clinicians and medical physicists to deliver the treatment in a much faster and precise manner. ${ }^{9}$ The practice of SRS is expanding every day, and attempts are being made to expand it to many more indications of intracranial lesions. With the advancement in early diagnosis, the future of SRS and SRT looks brighter.

With the vision to provide high-quality treatment to the people of Bangladesh, Evercare Hospital has established a state-of-the-art SRS/SRT facility with specialized manpower. To date, we have treated more than 35 patients, and their clinical outcomes are appreciable. No more abroad travel is required for the people of this country to avail such high precision treatment and it is available in their vicinity.

\section{Conflict of Interest}

None declared.

\section{Acknowledgments}

The authors would like to express their sincere gratitude to the management of Evercare Hospitals Dhaka for their continuous encouragement and co-operation in establishing the state-of-the-art facility. They would also like to thank their neurology and radiology department colleagues for their support.

\section{References}

1 Hussain SA, Sullivan R. Cancer control in Bangladesh. Jpn J Clin Oncol 2013;43(12):1159-1169

2 Hussain SM. Comprehensive update on cancer scenario of Bangladesh. South Asian J Cancer 2013;2(4):279-284

3 Gigliotti MJ, Hasan S, Liang Y, Chen D, Fuhrer R, Wegner RE. A 10-year experience of linear accelerator-based stereotactic radiosurgery/radiotherapy (SRS/SRT) for paraganglioma: a single institution experience and review of the literature. J Radiosurg SBRT 2018;5(3):183-190

4 Benedict SH, Bova FJ, Clark B, et al. Anniversary paper: the role of medical physicists in developing stereotactic radiosurgery. Med Phys 2008;35(9):4262-4277

5 Liepa Z, Auslands K, Apskalne D, Ozols R. Initial experience with using frameless image-guided radiosurgery for the treatment of brain metastases. Exp Oncol 2012;34(2):125-128

6 Rieber J, Tonndorf-Martini E, Schramm O, et al. Establishing stereotactic body radiotherapy with flattening filter free techniques in the treatment of pulmonary lesions - initial experiences from a single institution. Radiat Oncol 2016;11:80

7 Duan X, Giles W, Kirkpatrick JP, Yin FF. The effect of setup uncertainty on optimal dosimetric margin in LINAC-based stereotactic radiosurgery with dynamic conformal arc technique. J Radiosurg SBRT 2019;6(1):55-65

8 Solberg TD, Balter JM, Benedict SH, et al. Quality and safety considerations in stereotactic radiosurgery and stereotactic body radiation therapy: executive summary. Pract Radiat Oncol 2012;2(1):2-9

9 Sahgal A, Ma L, Chang E, et al. Advances in technology for intracranial stereotactic radiosurgery. Technol Cancer Res Treat 2009;8(4):271-280 